undergoing venesection, nowadays he is lucky if he gets away without a blood transfusion. Bernard Aschner's strange book is not only an attempt to justify much of the 'devil-exorcising' therapy, bleeding, cupping, purging and the like, of the eighteenth century orgies, but also to belittle the methods of modern scientific medicine and to reestablish some of the old. There is a grain of truth in his criticisms of modern medical trends with their failure to stem the rising tide of psychosomatic and degenerative disease, which he exploits to the utmost in the early parts of the book. It is difficult to see for whom the book was intended.

J.M.N.

\section{ATLAS OF BACTERIOLOGY}

By R. Cranston Lo'w and T. C. Dodns. E. \& S. Livingstone, Ltd. (Pp. vii and 168. 168 illustrations, 32s. 6d.)

As stated in the preface, this book is intended primarily for the medical undergraduate, and is to be used in conjunction with any standard textbook. It is beautifully produced, but the quality has made the cost high for a book which is only to illustrate what the student should see in the practical class. Many of the illustrations are extremely good, but a few are not of this high standard. The system adopted is useful and many fungi are included. This book should also prove useful to those who have to lecture in bacteriology to nurses and have not the necessary facilities for providing specimens.

S.D.N.

\section{SENSORY MECHANISMS OF THE RETINA}

\section{By Ragnar Granit, M.D. Oxford University}

Press, 35s.

In this book, Professor Granit sets out to give as complete an account as possible of the changes in the retina, from the absorption of light by the retinal pigments to the setting up of impulses in the fibres of the optic nerve. His review of the analysis of the breakdown of visual purple is a clear and comprehensible account of what is usually a confused and confusing subject. He has investigated the production of repetitive firing in nerve fibres under the influence of slowly rising currents, in order to extend our understanding of repetitive firing in sensory nerve endings under natural stimulation. The attempt is ingenious and has provided new information, but Hodgkin's work, which has appeared since this book was written, provides a much more elegant account of the spacing of impulses in terms of a local response leading up to the initiation of each impulse in the series.

The most valuable part of this book is a description of the results of five years' intensive work on the unitary mechanisms of the retina concerned with colour vision. The activity in single nerve fibres has been picked up by a microelectrode from the inner surface of the retina in a variety of vertebrate eyes. By stimulating with light of known intensity and wavelength, the spectral sensitivity of these units can be mapped out. Granit has obtained evidence for two mechanisms with a wide range of spectral sensitivity, called the photopic and scotopic dominators, and said to be responsible for sensations of luminosity during light and dark adaptation respectively. In addition there are six or seven mechanisms which are sensitive to much narrower spectral bands, two in the red, two in the blue, and two or three in the green.

It is impossible to fail to admire the neatness of the methods used and the physiological difficulties overcome. This kind of work is however far more difficult to interpret than studies on sensory receptors in the skin, since there is in this case an outlying part of the nervous system interposed between the sensory receptor cells and the first point at which one can pick up their impulses. With nearly all sensory mechanisms, the endings are inaccessible, and the stimulus used may be distorted by the intervening tissue. Once the impulse is set up, it is transmitted along the nerve fibre in which it can be detected, and although peripheral nerve fibres may supply a few endings of the same type, interference in the pattern of impulses by two active endings connected to the same fibre is surprisingly rare. In the eye, the difficulties are reversed. If the cornea and lens are removed, the stimulus reaches the retina without modification. The lateral connections which occur between ganglion cells ensure that even at the macula one nerve fibre can be excited by a considerable number of cones. In the periphery, Hartline has shown that the receptive field of a single fibre extends up to I $\mathrm{mm}$. in diameter.

It is not therefore surprising that the spectral sensitivity curves are often complex, and that they change with changing conditions of dark adaptation. The scotopic dominator, whose sensitivity curve agrees exactly with the absorption curve of visual purple can hardly be anything but a rod. Whether the other mechanisms described are in fact the ultimate units of retinal activity does not seem to be quite certain.

What is certain is that there is good reason for modifying the Young-Helmholtz three colour theory. If a photopic dominator exists, and is responsible for sensations of luminosity, then it is no longer surprising that dichromats have a sensi tivity to luminosity indistinguishable from that of normal individuals. Anomalous trichromatism is also more easily accounted for on the new theory. This book should encourage the search for more evidence of the nature of the ultimate units of retinal activity. It is invaluable to all who are interested in the physiology of vision, in dark adaptation and colour blindness.

It is a pity that the publishers who have provided good printing and paper in spite of the present difficulties, should have to allow this book to appear in such a shoddy binding.

D.W. 